\title{
Variabilidade climática e a modelagem ecológica da Biomphalaria glabrata: cenários futuros $(2050$ e 2070$)$ para o hospedeiro intermediário da esquistossomose no Brasil
}

\author{
Márcia Eliane Silva Carvalho ${ }^{(a)}$, Francisco de Assis Mendonça ${ }^{(b)}$, Sindiany Suelen Caduda dos \\ Santos ${ }^{(c)}$ \\ (a) Departamento de Geografia, Universidade Federal de Sergipe, Email: marciacarvalho_ufs@ yahoo.com.br \\ (b) Departamento de Geografia, Universidade Federal do Paraná, Email:chico@ufpr.br \\ (c) Departamento de Biologia, Universidade Federal de Sergipe, Email: sindianyscs@ gmail.com
}

\section{Eixo 3: Climatologia em diferentes níveis escalares: mudanças e variabilidades}

Resumo

\begin{abstract}
A variabilidade climática em curso pode gerar mudanças futuras significativas na distribuição de várias espécies, pois podem afetar o seu nicho e habitat. Este artigo tem como objetivo analisar a influência de variáveis ambientais na distribuição potencial futura (+50 anos e +70 anos) do hospedeiro intermediário da esquistossomose (Biomphalaria glabrata) através da modelagem ecológica utilizando o programa MAXENT. Ao analisar o modelo de distribuição futura de 50 anos, a amplitude térmica anual e a precipitação anual apresentaram-se como o maior percentual de contribuição. Para projeção futura de 70 anos, a temperatura média do trimestre mais frio, apresentou o maior percentual de contribuição para a distribuição da espécie no ambiente. A análise realizada expressa a importância da ampliação dos estudos sobre a variabilidade climática e sua relação com a saúde, poisrevalidou que na região Nordeste do Brasil encontram-se os pontos quentes de ocorrência da B. glabrata, com cenário de distribuição potencial para outros estados brasileiros.
\end{abstract}

Palavras chave: Variabilidade climática, Modelagem Ecológica, Esquistossomose

\section{1. Introdução}

A variabilidade climática em curso pode gerar mudanças futuras significativas na distribuição de várias espécies, pois podem afetar o seu nicho e habitat. Tal situação torna-se foco de várias pesquisas que visam compreender como estas variações podem repercutir sobre a distribuição espacial de espécies transmissoras de doenças para os seres humanos.

Assim, os estudos sobre a variabilidade climática tem-se destacado na atualidade com maior ênfase em virtude do aquecimento da atmosfera do planeta Terra, cujos efeitos e impactos em tempo presente e futuro ainda não estão totalmente esclarecidos (MENDONÇA, 2003).

Atualmente, a modelagem de nicho ecológico, uma ferramenta computacional, apresenta cenários preditivos para o comportamento de várias espécies. Trata-se de uma técnica que combina variáveis, 
projetando cenários que identifica as regiões potencialmente apropriadas para a ocorrência da espécie pesquisada, criando um mapa de distribuição potencial.

Os modelos de distribuição geográfica potencial de espécies surgiram com a proposta de preencher as lacunas de conhecimento sobre os limites geográficos de espécies de interesse, ajudando a entender quais mecanismos influenciam na distribuição com grande relação com as variáveis ambientais e em especial com as climáticas (GIANNINI, et al., 2012).

A modelagem de nicho apresenta então diversas aplicações. Dentre elas, pode citar: identificar espécies que poderiam ser utilizadas em trabalhos de recuperação ambiental; avaliar o potencial de ameaça de espécies invasoras; auxiliar na determinação de áreas prioritárias para conservação; estudar possíveis rotas de disseminação de doenças infecciosas, simulando, assim, cenários futuros e alternativos dando suporte para a tomada de decisão pelo poder público, minimizando gastos e reduzindo esforços (ALMEIDA JÚNIOR, 2010).

Desta forma, buscar compreender como as consequências das alterações climáticas em âmbito global / local repercutirão de forma direta ou indireta sobre a disseminação de doenças e consequente repercussão sobre a saúde humana, torna-se elemento fundamental nas pesquisas da atualidade (MENDONÇA, 2003).

No âmbito desta discussão, dentre as doenças infecciosas, destacam-se as de veiculação hídrica, pois ainda prevalecem no cenário das regiões urbanas e periurbanas, causando elevada morbidade no Brasil.

Várias destas doenças têm sua origem e mecanismos associados a determinadas espécies que agem como vetores ou hospedeiros, como a febre amarela, malária, dengue, amebíase, cólera, micoses, leptospirose, esquistossomose, dentre outras parasitose, que acomete, em muitos casos, a população mais vulnerável do ponto de vista socioeconômico (CONFALONIERI, 2003; MENDONÇA, 2005; BARCELLOS, 2009).

O Schistosoma mansoni, causador da esquistossomose, parasitose humana mais disseminada no mundo (OMS, 2010) e considerada uma problemática de saúde pública no Brasil, é um helminto que necessita da participação de um hospedeiro intermediário para completar seu ciclo reprodutivo, infectando posteriormente os seres humanos.

No Brasil, os hospedeiros intermediários de Schistosoma mansoni são as espécies de caramujo Biomphalaria glabrata, B. straminea, B. tenagophila (BRASIL, 2008). Estabelecida inicialmente no litoral, a esquistossomose expandiu-se amplamente pelo Brasil, dada as condições climática favoráveis ao estabelecimento e reprodução do hospedeiro intermediário, bem como pela precariedade nas condições de saneamento que persistem até a atualidade. 
XVII Simpósio Brasileiro

de Geografia Física Aplicada

I Congresso Nacional

de Geografia Física
OS DESAFIOS DA GEOGRAFIA FÍSICA NA FRONTEIRA DO CONHECIMENTO

Instituto de Geociências - Unicamp

Campinas - SP

28 de Junho à 02 de Julho de 2017

Considerando a importância para a saúde pública do estudo da distribuição espacial da B. glabrata e dos incipientes estudos sobre a modelagem desta espécie, este artigo tem como objetivo analisar a influencia de variáveis ambientais na distribuição potencial futura (+50 anos e +70 anos) do hospedeiro intermediário da esquistossomose através da modelagem ecológica.

\section{Procedimentos Metodológicos:}

Para atingir o objetivo proposto foi utilizado o programa MAXENT, considerado como o de melhor desempenho em termos de distribuição potencial das espécies, tomando como base a proposta metodológica de Santos et.al. (2015).

Como requisito inicial para utilizar o MAXENT faz-se necessário a identificação correta da espécie e montagem do banco de dados com o registro das coordenadas geográficas de localização da espécie em tempo presente. Em seguida, deve-se selecionar as variáveis ambientais.

As variáveis ambientais utilizadas foram coletadas no banco de dados do Instituto Nacional de Pesquisas Espaciais (AMBDATA, 2015) e no Worldclim (HIJMANS, et.al., 2005) que utiliza as projeções do quinto relatório do IPCC. Foram selecionadas as variáveis que, de acordo com a literatura, apresentam relação direta com a distribuição da B. glabrata: temperatura média anual, sazonalidade da temperatura, amplitude térmica anual, temperatura média do trimestre mais seco, temperatura média do trimestre mais quente, temperatura média do trimestre mais frio, precipitação anual, sazonalidade da precipitação, precipitação do trimestre mais chuvoso, precipitação do trimestre mais seco, precipitação do trimestre mais quente. Além destas variáveis climáticas foi também considerada a densidade de drenagem pela relação da espécie com o ambiente aquático.

Como indicador de qualidade do modelo, a partir dos valores de AUC ("AreaUnder The Curve"), utilizouse a seguinte escala (METZ, 1986, apud GIANNINI, et.al., 2012): excelente $(0,9-1)$; bom $(0,8-0,9)$; médio $(0,7-0,8)$; ruim $(0,6-0,7)$; muito ruim $(0,5-0,6)$.

Como banco de dados de referência da espécie pesquisada foram utilizados os dados de Carvalho et.al. (2008) que apresenta uma listagem atualizada de ocorrência da B. glabrata para todo o país.

\section{Cenários de distribuição potencial futura para a Biomphalaria glabrata e sua relação com a variabilidade climática:}

No Brasil B. glabrata foi notificada em 16 estados brasileiros: Alagoas, Bahia, Espírito Santo, Goiás, Maranhão, Minas Gerais, Para, Paraíba, Paraná, Pernambuco, Piauí, Rio Grande do Norte, Rio Grande do 
Sul, Rio de Janeiro, São Paulo e Sergipe, além do Distrito Federal. No total abrange 809 municípios (Figura 01) (CARVALHO et.al., 2008).

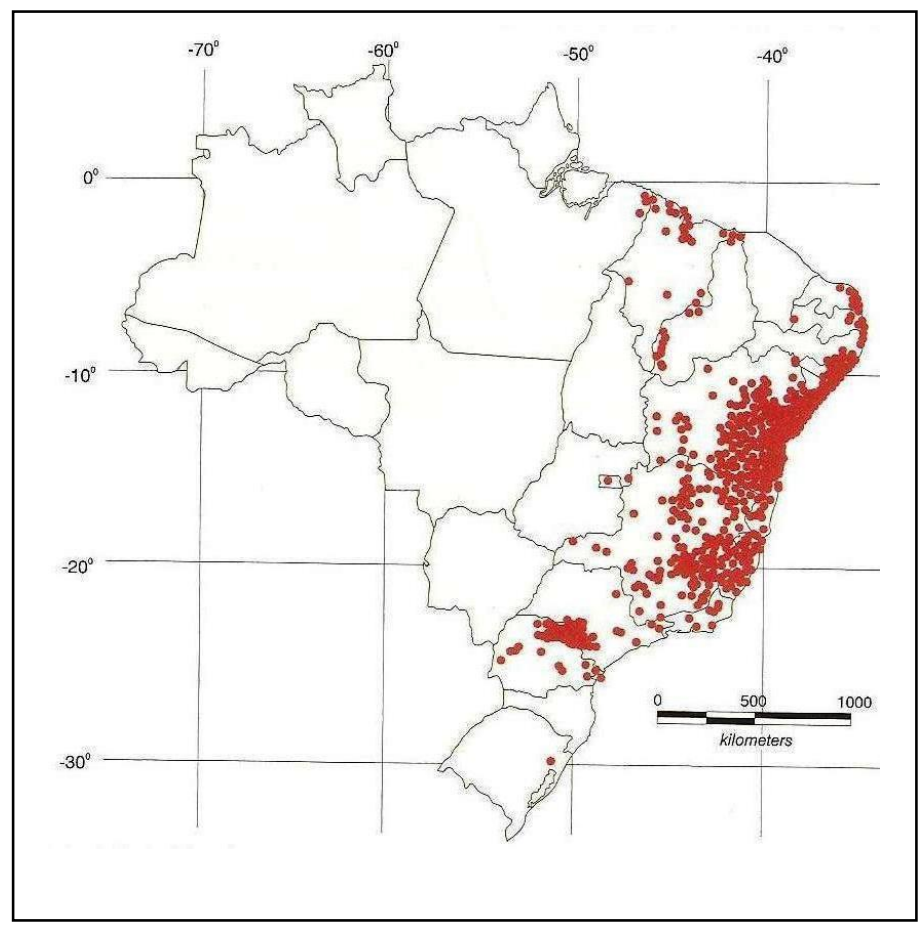

Figura 01. Distribuição espacial do hospedeiro intermediário da esquistossomose, Biomphalaria glabrata, por municípios no Brasil. Fonte: CARVALHO et al., 2008.

O estudo comportamental da Biomphalaria glabrata embora recomendado pela Organização Mundial de Saúde desde seus primeiros relatórios sobre a esquistossomose, não foram realizados de maneira aprofundada. A espécie apresenta uma série de comportamentos adaptativos que a permitem sobreviver após o uso de moluscicidas no ambiente, repovoando-o rapidamente em função de poderem sobreviver fora da água em estágio de dormência, de serem prolíferos e hermafroditas com capacidade de autofecundação (BARBOSA, 1995).

Vale ressaltar que a importância epidemiológica da espécie Biomphalaria glabrata está relacionada pelo fato dela ser considerada como uma das mais importantes vetores da esquistossomose no litoral do Nordeste (SOUZA et al., 2010).

Na tabela 01 consta o percentual de contribuição de cada variável no processo de distribuição da espécie em diferentes tempos, demonstrando a importância dos condicionantes climáticos no processo de distribuição espacial e temporal da espécie em estudo. 
XVII Simpósio Brasileiro

de Geografia Fisica Aplicada

I Congresso Nacional de Geografia Física
OS DESAFIOS DA GEOGRAFIA FÍSICA NA FRONTEIRA DO CONHECIMENTO

Instituto de Geociências - Unicamp

Campinas - SP

28 de Junho à 02 de Julho de 2017

Tabela 01 - Percentual de contribuição das variáveis na distribuição potencial da espécie B. glabrata no tempo futuro.

\begin{tabular}{c|c|c|c}
\hline Variável* & Definição & Futuro +50 & Futuro +70 \\
\hline Bio4 & Sazonalidade da temperatura & $\mathbf{3 , 6}$ & $\mathbf{2}$ \\
\hline Bio7 & Amplitude térmica anual & $\mathbf{3 0 , 5}$ & $\mathbf{2 1 , 6}$ \\
\hline Bio 12 & Precipitação anual & 15,6 & $\mathbf{6 , 7}$ \\
\hline Bio 17 & Precipitação do trimestre mais seco & $\mathbf{3}$ & $\mathbf{4 , 7}$ \\
\hline Alt. & Altitude & - & - \\
\hline Decliv. & Declividade & - & - \\
\hline Bio 11 & Temperatura média do trimestre mais frio & 26 & 47 \\
\hline Bio5 & Temperatura máxima do mês mais quente & - & - \\
\hline Bio9 & Temperatura média do trimestre mais & 1,7 & 2,4 \\
& seco & 16,1 & 1 \\
\hline Bio1 & Temperatura média anual & - & - \\
\hline Dens.Dren & Densidade da rede de drenagem & - & 1,1 \\
\hline Bio 10 & Temperatura média do trimestre mais \\
& quente & 0,4 & 2,9 \\
\hline Bio 16 & Precipitação do trimestre mais chuvoso & 1,3 & 1,9 \\
\hline Bio 18 & Precipitação do trimestre mais quente & $\mathbf{1 , 9}$ & $\mathbf{8 , 6}$ \\
\hline Bio 15 & Sazonalidade da precipitação & $\mathbf{0 , 9 5 8}$ & \\
\hline AUC & AreaUnder The Curve (que valida o & & \\
& modelo) & & \\
\hline
\end{tabular}

* Dados disponíveis no banco de dados do Instituto Nacional de Pesquisas Espaciais (AMBDATA, 2015) e do Worldclim (HIJMANS et al., 2005).

Analisando o AUC (curva gerada pelo Maxent que determina a validade do modelo) para os modelos gerados, constata-se que todos apresentaram valores que se aproxima da unidade, o que significa que o modelo apresentou alto desempenho: futuro 50 anos - 0,958 e futuro 70 anos $-0,952$.

Ao analisar o modelo de distribuição futura de 50 anos, a amplitude térmica anual e a precipitação anual apresentaram-se como o maior percentual de contribuição. Para projeção futura de 70 anos, a temperatura média do trimestre mais frio, será o fator que apresenta o maior percentual de contribuição para a distribuição da espécie no ambiente, considerando as mudanças climáticas em curso.

Em tempo presente os pontos quentes de ocorrência concentram-se na região litorânea do nordeste brasileiro, com destaque na Paraíba, Alagoas, Sergipe e Bahia, além de parte de Minas Gerais, Espírito Santo e Rio de Janeiro.

Ao analisar os modelos em tempo futuro 50 anos, constata-se a manutenção dos pontos quentes de distribuição potencial da espécie na região Nordeste e com distribuição potencial média ampliada para o estado de Mato Grosso do Sul e em menor escala no norte de Roraima, ambos os estados não endêmicos para a esquistossomose (Figura 02). 


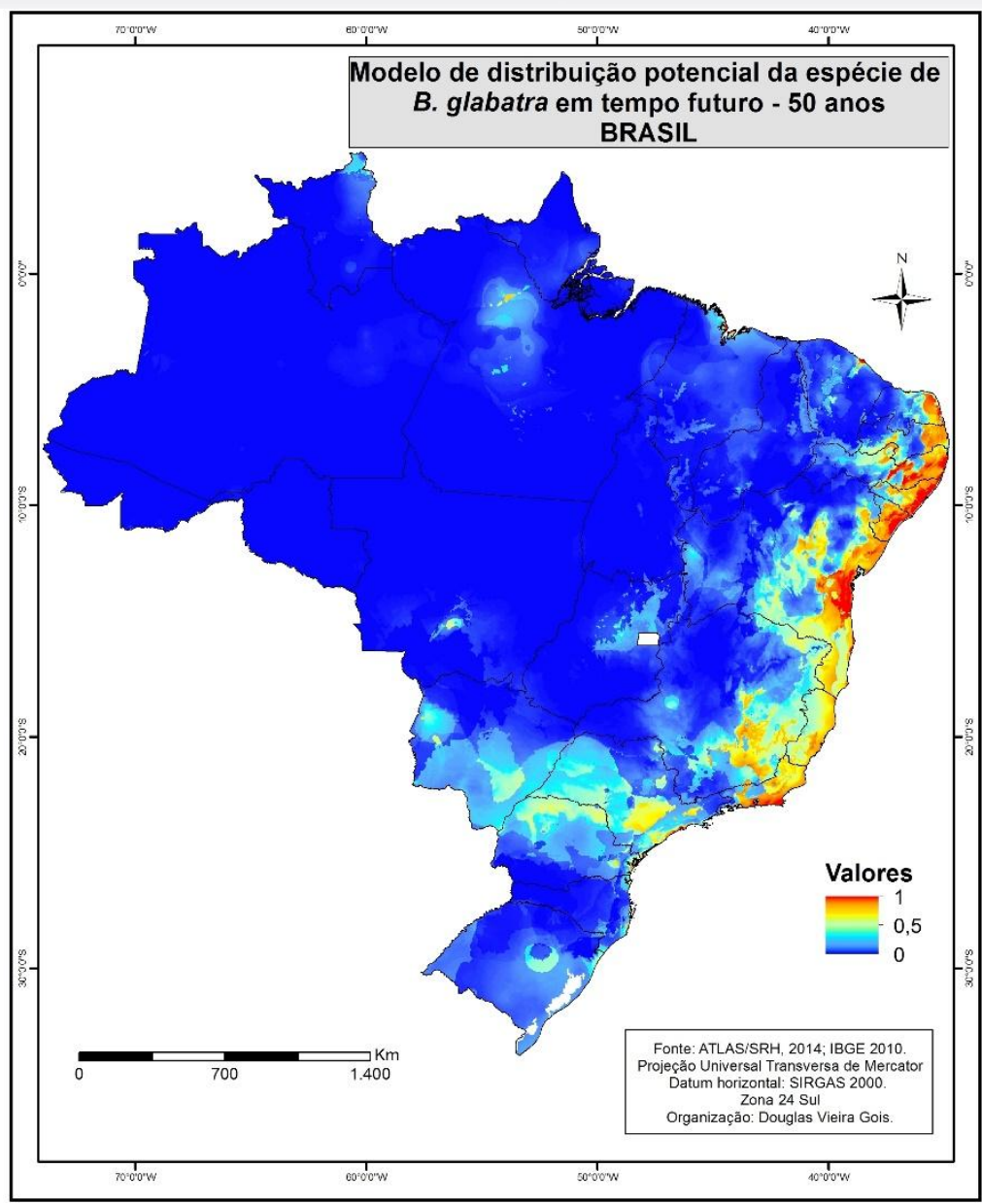

Figura 02 - Modelo de distribuição potencial da espécie de B. glabrataem tempo futuro (50 anos). Organizado pelos autores.

Considerando as variáveis climáticas que apresentaram maior peso no percentual de contribuição (amplitude térmica anual e a precipitação anual), considerando as exigências ecológicas da espécie, que não tolera elevadas temperatura e nem redução excessiva da pluviosidade, e considerando as projeções do IPCC sobre aumento da temperatura média global nos próximos 50 anos e redução da precipitação em determinadas áreas do Brasil, variações mais drásticas nestas variáveis podem ser as responsáveis pela redução na área de ocorrência potencial da espécie, reduzindo os pontos quentes de ocorrência na região sudeste, mas ampliando em alguns casos, os pontos quentes em outros estados do Nordeste do Brasil.

Considerando a forte associação da espécie em estudo com a expansão da esquistossomose (Figura 03), Chieffi e Waldman (1988) apontam que na região norte do país a esquistossomose não tem exercido papel destacado como problema de saúde pública, embora na década de 40, foram descritos os primeiros focos de transmissão autóctone de esquistossomose no Pará. Entretanto, Atualmente na região Amazônica a preocupação deve ser premente em virtude da possiblidade do surgimento de novos focos, relacionados à 


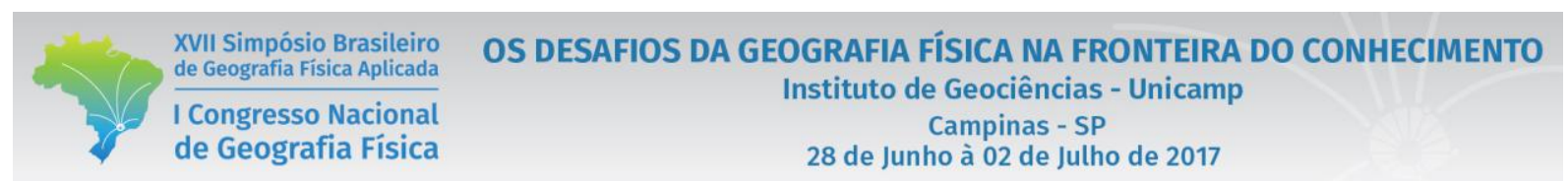

abertura de estradas, empreendimentos agropecuários e à instalação de usinas hidrelétricas, cujos fluxos migratórios podem ampliar as áreas indenes e/ou foco para endêmicas.

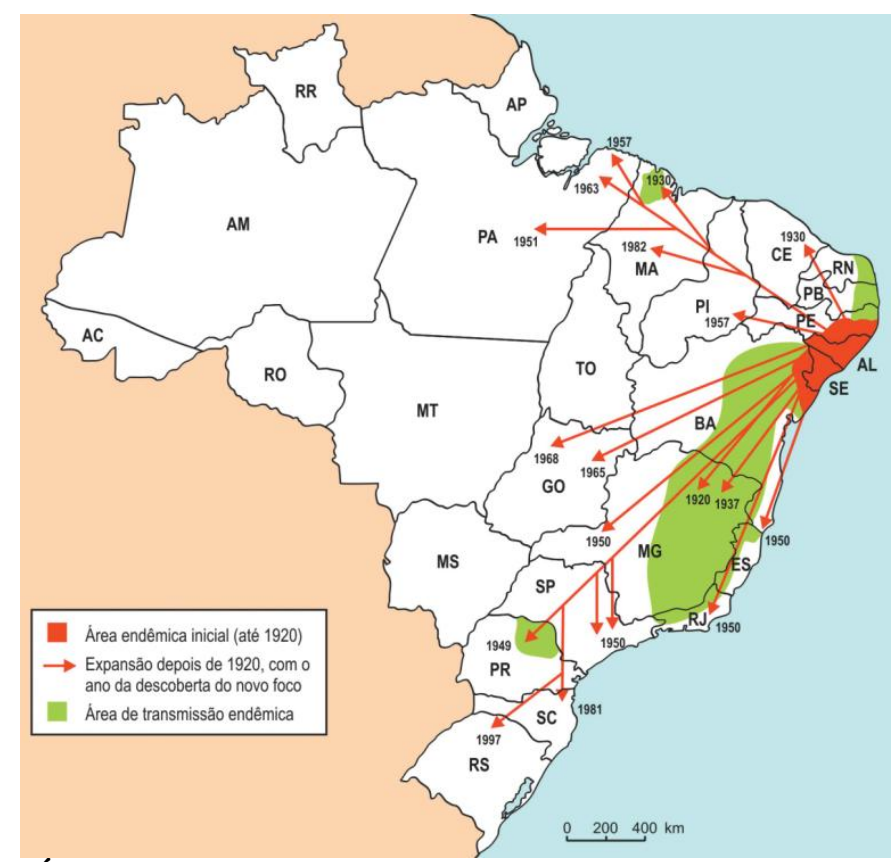

Figura 03 - Área endêmica para a esquistossomose e área de expansão após 1920.

Fonte: BRASIL, 2014.

No período futuro de 70 anos, nota-se pela análise do modelo, comparando com o modelo de futuro 50 anos, que possivelmente em função de processos adaptativos e/ou a presença de excesso de matéria orgânica no ambiente associada com a ausência de saneamento adequado que o Nordeste amplia os pontos quentes de ocorrência da espécie, bem como possibilidade de ampliação de possível área de ocorrência na região norte do país e ratifica a possível área de distribuição ampliada para o Mato Grosso do Sul e Paraná (Figura 04). 


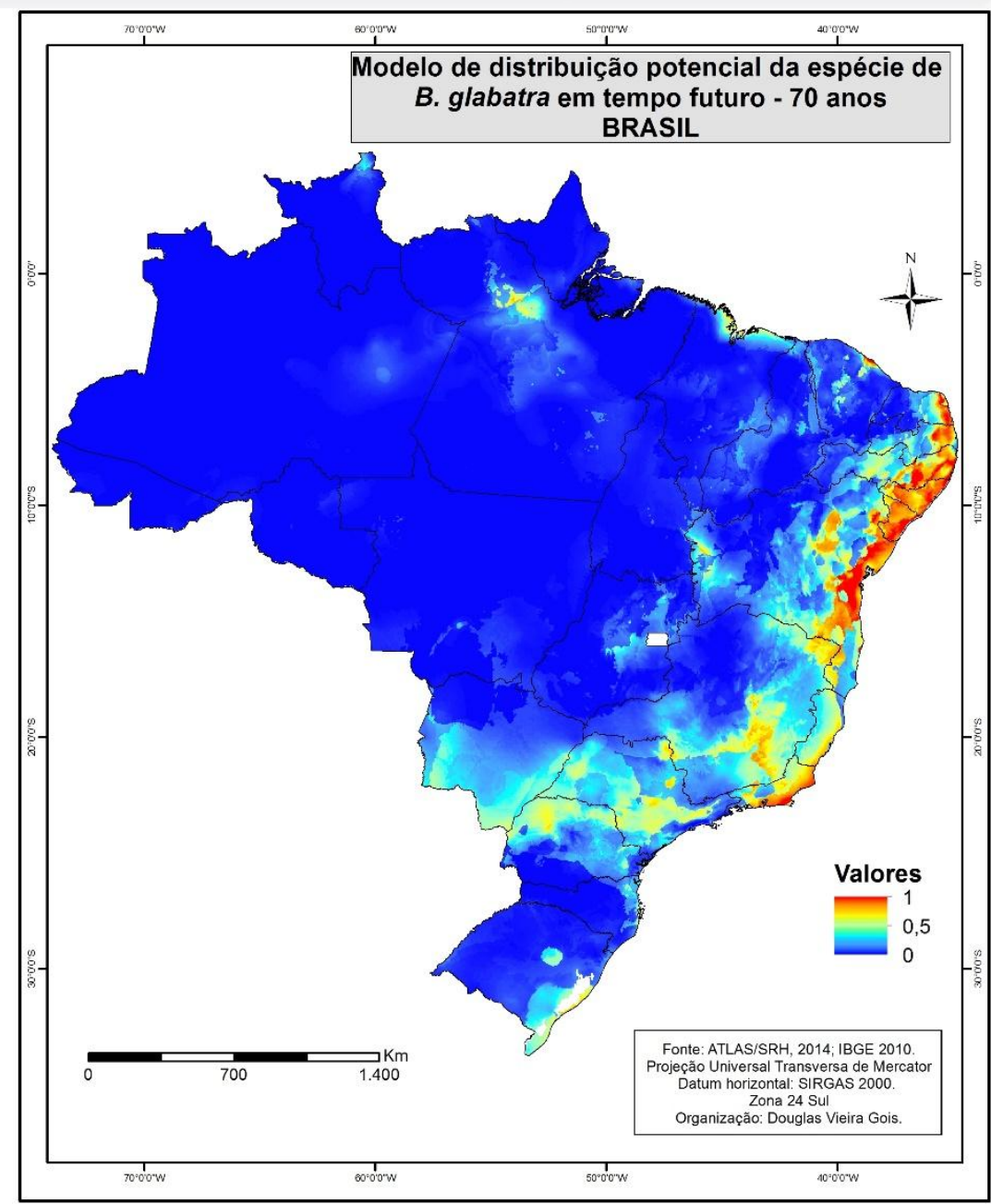

Figura 04 - Modelo de distribuição potencial da espécie de $B$. glabrata em tempo futuro (70 anos). Organizado pelos autores.

Esta análise ratifica pesquisas sobre aos atributos abióticos associados a reprodução e distribuição da $B$. glabrata. De acordo com Gomes (2011), a temperatura ideal para o desenvolvimento deste molusco varia de 20-26 ${ }^{\circ} \mathrm{C}$, podendo tolerar temperaturas que variam de 18 a $41{ }^{\circ} \mathrm{C}$ (BRASIL, 2008), demonstrando a sua plasticidade e capacidade adaptativa a essas variações.

Para além das variações climáticas que apresentam repercursão sobre a distribuição desta espécie, mudanças comportamentais da $B$. glabrata associadas à ocupação desordenada e a ausência de saneamento em áreas urbanas vêm sendo o principal causa da dispersão da esquistossomose no Brasil (PAZ, 1997; ARAÚJO, 2007; VASCONCELOS, 2009; OLIVEIRA, 2011; TIBIRIÇA, 2011; TEIXEIRA, 2011). Havendo condições propicias para sua dispersão e reprodução, rapidamente elas povoam o ambiente. Decorre então deste fato, a importância de identificar áreas potenciais de dispersão da espécie, 
XVII Simpósio Brasileiro

de Geografia Fisica Aplicada

I Congresso Nacional

de Geografia Física
OS DESAFIOS DA GEOGRAFIA FÍSICA NA FRONTEIRA DO CONHECIMENTO

Instituto de Geociências - Unicamp

Campinas - SP

28 de Junho à 02 de Julho de 2017

pois uma vez estabelecida tanto no meio rural quando no urbano, têm-se as condições propicias também para a dispersão da esquistossomose no território brasileiro.

Outro fator abiótico importante que influenciam a densidade do caramujo vetor é a pluviometria, pois determina a ocorrência e ampliação dos criadouros de Biomphalaria (GOMES, 2011), disseminando a espécie em períodos mais chuvosos para outras localidades.

O modelo de distribuição potencial aponta cenários futuros de possíveis áreas potenciais de distribuição e intensificação dos pontos quentes de ocorrência no Nordeste do Brasil. Os modelos encerram possibilidades de ocorrência e demonstram possíveis mudanças no cenário climático que podem gerar novas áreas de ampliação de ocorrência da B. glabrata. Estes dados podem ser tomados como referência e base para estudo mais específicos no âmbito da saúde pública dado ao fato de que a ocorrência desta espécie está intimamente relacionada aos focos de esquistossomose.

\section{Considerações Finais}

Vale considerar que com o cenário de aumento da temperatura da Terra e redução da precipitação, vários estudos ainda necessitam aprofundar se estas mudanças irão causar impacto direto ou indireto sobre a ecologia, nicho e habitat de várias espécies.

$\mathrm{Na}$ análise realizada, o modelo revalidou a região Nordeste do Brasil como pontos quentes de ocorrência da espécie B. glabrata, mesmo considerando as alterações climáticas futuras,o que associado a vulnerabilidades socioambientais ratificam a necessidade de políticas públicas que efetivem e universalizem o saneamento ambiental e melhoria nas condições de vida da população visando que tal espécie não seja unicamente relacionada com a transmissão da esquistossomose.

O conhecimento dos cenários futuros de distribuição potencial da espécie de Biomphalaria glabrata demonstrou também que há condições potenciais da espécie ampliar sua área de abrangência para os estados do Mato Grosso do Sul e Roraima que são considerados como não endêmicos para a esquistossomose, bem como redução para o estado do Paraná.

Tal estudo torna-se importante do ponto de vista epidemiológico,pois permite a identificação das áreas com risco de instalação de novos focos de transmissão da doença e/ou ampliação dos existentes, fornecendo subsídios aos serviços de saúde no que tange as ações de controle e vigilância desta endemia.

Com o esclarecimento do ciclo epidemiológico esquistossomose e sua relação direta com as espécies de Biomphalaria no Brasil, os estudos de modelagem ecológica podem ampliar o conhecimento 
comportamental das espécies hospedeiras a realidades diversas, sendo, portanto, de utilidade pública no que se refere a estratégias de combate a esta enfermidade.

Além do mais, a espécie em estudo tem alta capacidade adaptativa e se associada a condições ambientais de degradação ambiental encontram solo fértil para se reproduzir e em condições ecoepidemiológicas adequadas podem ampliar o ciclo reprodutivo da esquistossomose.

Vale lembrar que modelos são uma tentativa de reprodução da realidade, devendo ser considerados que processos adaptativos, mudanças nas estruturas sociais e econômicas podem trazer mudanças significativas nas projeções. Assim, vários elementos institucionais devem atuar em conjunto no processo de erradicação da doença, sendo que o uso da modelagem e de técnicas de geoprocessamento possam ser contributos para entender melhor os processos de saúde/doença voltando o olhar para a intrínseca relação entre ambiente/qualidade de vida/saúde coletiva.

\section{Bibliografia}

ALMEIDA JÚNIOR, E. B. de. 2010. Modelagem como ferramenta ecológica. Disponível em: http://oficinacientifica.com.br/index.php. Acesso em: 10/10/2016.

AMBDATA. Variáveis Ambientais para Modelagem de distribuição de espécies. Disponível em: http://www.dpi.inpe.br/Ambdata/descricao_dados.php. Acesso em: 10/12/2016.

ARAÚJO, K.C.G.M., et al. Análise espacial dos focos de Biomphalariaglabrata $e$ de casos humanos de esquistossomose mansônica em Porto de Galinhas, Pernambuco, Brasil, no ano 2000. Cadernos de Saúde Pública[online]. 2007, vol.23, n.2, pp.409-417.

BARBOSA, Frederico Simões (org). Tópicos em Malacalogia Médica. Rio de Janeiro: FIOCRUZ/Scielo Books. 1995.

BARCELLOS, Christovam et. al. Mudanças climáticas e ambientais e as doenças infecciosas: cenários e incertezas para o Brasil. Epidemiologia e Serviços de Saúde, Brasília, v. 18, n. 3, p. 285-304, jul-set. 2009.

BRASIL. Vigilância e controle de moluscos de importância epidemiológica: diretrizes técnicas. Programa de Vigilância e Controle da Esquistossomose (PCE) / Ministério da Saúde. 2. ed. Brasília: Editora do Ministério da Saúde. 2008.

BRASIL. Vigilância da Esquistossomose mansoni: diretrizes técnicas. 4. ed. Brasília: Ministério da Saúde, 2014.

CARVALHO, O. dos S. et.al. Importância epidemiológica dos moluscos do gênero Biomphalaria. In:Carvalho, O. dos Santos; et.al. Schistosoma mansoni: uma visão multidisciplinar. Rio de Janeiro: FIOCRUZ,2008, p. 309-346.

CHIEFFI, Pedro Paulo; WALDMAN, Eliseu Alves. Aspectos particulares do comportamento epidemiológico da esquistossomose mansônica no Estado de São Paulo, Brasil.Cadernos de Saúde Pública. Rio de Janeiro, v.4, n.3. Jul/Sept, 1988.

CONFALONIERI, Ulisses. E. C. Variabilidade climática, vulnerabilidades social e saúde no Brasil.Terra Livre. São Paulo, vol. 1, n. 20, p. 193-204, jan/jul, 2003 


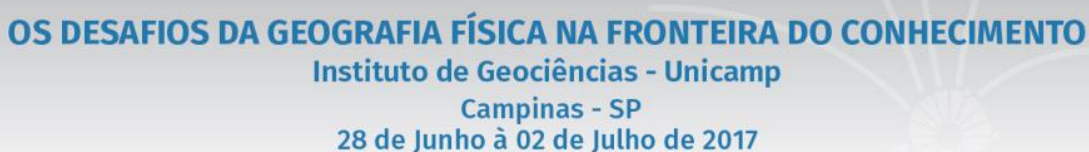

28 de Junho à 02 de Julho de 2017

GIANNINI, T.C. et al. Desafios atuais da modelagem preditiva de distribuição de espécies. Rodriguésia,v.63, n. 3, p. 733-749. 2012.

GOMES, Elaine C. de S. Modelo de risco para a esquistossomose. Tese de doutorado. Fundação Oswaldo Cruz, Recife, 2011.

HIJMANS, R.J., et al. Very high resolutioninterpolatedclimatesurfaces for global landareas. InternationalJournalofClimatology 25, p.1965-1978, 2005.

MENDONÇA, Francisco. Aquecimento global e saúde: uma perspectiva geográfica. Terra Livre. São Paulo, vol. I, n.20, 2003, p. 205-221.

Clima, tropicalidade e saúde: uma perspectiva a partir da intensificação do aquecimento global. Revista Brasileira de Climatologia, vol. 1, n. 1, 2005, p.100-112.

OLIVEIRA, Daniel Santos. Determinação e avaliação de focos esquistossomóticos em áreas urbanas do estado de Sergipe/Brasil. Dissertação de Mestrado em Saúde e Ambiente. Aracaju: Universidade Tiradentes 2011.

OMS. Trabalhando para superar o impacto global de doenças tropicais negligenciadas: Primeiro relatório da OMS sobre doenças tropicais negligenciadas. Organização Mundial de Saúde, 2010, 188p.

PAZ, R.J. da.Biologia e Ecologia deBiomphalariaglabrata(Say, 1818) (Mollusca: Pulmonata: Planorbidae), na Fazenda Árvore Alta, Alhandra (Paraíba: Brasil). Dissertação de Mestrado. João Pessoa: UFPB 1997.

SANTOS, S.S.C. et.al. Cenários de distribuição de mangues no litoral norte e nordeste brasileiro a partir da modelagem de distribuição potencial das espécies. Revista Okara, v.9, n.2, p.313-324, 2015.

TEIXEIRA, R. dos S.; et.al. Espacialização do Caramujo da espécie BiomphalariaGlabrata do córrego da Lagoa do Parque de Pituaçu Salvador/ BA. Candombá - Revista Virtual, v. 7, n. 1, p. 55-64, jan/dez, 2011.

TIBIRIÇÁ, S.H.C.; GUIMARÃES, F.B., TEIXEIRA, M.T.B. A esquistossomose mansoni no contexto da política de saúde brasileira.Cadernos de saúde coletiva. 16 (Supl.1), 2011, p. 1375-1381. 